\title{
ON THE RADIAL LIMITS OF ANALYTIC FUNCTIONS
}

\section{A. J. LOHWATER}

1. Examples have been given $[5$, p. 185] of functions $f(z)$, analytic in the unit-circle $K:|z|<1$, and not identically constant, for which the radial $\operatorname{limit} f\left(e^{i \theta}\right)=\lim _{r \rightarrow 1} f\left(r e^{i \theta}\right)$ is zero for all $e^{i \theta}$ on $|z|=1$ except for a set of linear measure zero. In view of the Riesz-Nevanlinna theorem [6, p. 197], such functions cannot be bounded, or even of bounded characteristic, in $|z|<1$. Functions of this sort appear again whenever we have an analytic function $f(z)$ whose radial limits coincide almost everywhere with the radial limits of a bounded analytic function $g(z)$, for the difference $F(z)=f(z)-g(z)$ has a radial limit zero almost everywhere on $|z|=1$. The Riesz-Nevanlinna theorem shows that, if $f(z)$ is bounded, or of bounded characteristic, and if the radial limit values of $f(z)$ coincide almost everywhere on an arc of $|z|=1$ with the radial limit values of $g(z)$, then $F(z)$ must be identically zero in $|z|<1$. The object of this note is to discuss certain aspects of the behavior of nonconstant analytic functions whose radial limits vanish almost everywhere on an arc $A\left(\theta_{1}<\theta<\theta_{2}\right)$ of $|z|=1$. One result of such a study (which the author plans as a sequel to this note) will be to give some idea of the way in which a function $f(z)$, whose radial limits coincide almost everywhere with the radial limits of a function $g(z)$ of bounded characteristic, can differ from $g(z)$.

We shall say that a nonconstant function $f(z)$, analytic in $|z|<1$, is of class $(L P)$ on an arc $A$ of $|z|=1$, if $\lim _{r \rightarrow 1} f\left(r e^{i \theta}\right)=f\left(e^{i \theta}\right)=0$ for almost all $e^{i \theta}$ belonging to the arc $A$. If the $\operatorname{arc} A$ is the whole circumference $|z|=1$, we shall say simply that the function $f(z)$ is of class (LP).

One property of functions which are of class (LP) on an arc $A$ is immediate: the cluster set of $f(z)$ at each point $e^{i \theta_{0}}$ of $A$ (i.e., the set of all values $\alpha$ with the property that there exists a sequence $\left\{z_{n}\right\},\left|z_{n}\right|$ $<1, \lim _{n \rightarrow \infty} z_{n}=e^{i \theta_{0}}$, such that $\left.\lim _{n \rightarrow \infty} f\left(z_{n}\right)=\alpha\right)$ is the whole plane. For, if there is a point $e^{i \theta_{0}}$ on $A$ and a complex number $\alpha$ which does not belong to the cluster set of $f(z)$ at $e^{i \theta}$, then there is a circular neighborhood $V\left(e^{i \theta_{0}}\right)$ of $e^{i \theta_{0}}$ such that, in $V\left(e^{i \theta_{0}}\right) \cap K$, the function $g(z)$ $=[f(z)-\alpha]^{-1}$ is analytic and bounded. Since the function $g(z)$ has the constant limit $-1 / \alpha$ along almost all normal segments drawn to that arc of $|z|=1$ which bounds $V \cap K$, it follows from a simple corollary of the Riesz-Nevanlinna theorem that $g(z)$, and hence $f(z)$,

Presented to the Society, February 27, 1954; received by the editors April 6, 1954 and, in revised form, May 24, 1954. 
must be identically constant in $V \cap K$ and, a fortiori, in $|z|<1$. This property, i.e., that the cluster set is the whole plane, sometimes called the Weierstrass property, suggests that we investigate the values $\zeta$ which $f(z)$ admits as asymptotic values, i.e., the values to which $f(z)$ tends as $z$ approaches a point $P$ of $|z|=1$ along a curve terminating at $P$. We shall show (Theorem 1 ) that every complex value $\zeta$ (including $\infty$ ) is an asymptotic value of a function $f(z)$ of class (LP) provided that the $\zeta$-points, i.e., the points $z_{k}$ for which $f\left(z_{k}\right)=\zeta$, satisfy the condition

$$
\sum_{k=1}^{\infty}\left(1-\left|z_{k}\right|\right)<\infty .
$$

In Theorem 2 we show that a function of class (LP) on an arc $A$ admits every complex number $\zeta$ as an asymptotic value in every neighborhood of every point $e^{i \theta}$ of $A$ if the $\zeta$-points in some neighborhood $V\left(e^{i \theta}\right) \cap K$ of $e^{i \theta}$ satisfy (1). Theorem 2 then contains Theorem 1, but the proof of Theorem 1 is considerably simpler, and we give a separate proof.

Lemma 1. Let $f(z)$ be analytic and different from 0 in $|z|<1$, and let the modulus $\left|f\left(r e^{i \theta}\right)\right|$ have radial limit 1 for almost all $e^{i \theta}$ on $|z|=1$. Then unless $f(z)$ is identically constant in $|z|<1$, there exists a Jordan arc $\mathcal{L}$, lying in $|z|<1$ and terminating at a point $e^{i \theta_{0}}$ of $|z|=1$, such that, as $z \rightarrow e^{i \theta_{0}}$ along $\mathcal{L}$, either $f(z) \rightarrow 0$ or $f(z) \rightarrow \infty$. If there exists no path along which $f(z) \rightarrow 0$, then $|f(z)|>1$ in $|z|<1$.

Lemma 1 is equivalent to Theorems 5 and 6 of [3], and its proof is omitted here. For brevity, we shall say that a function which is analytic in $|z|<1$ and whose modulus $\left|f\left(r e^{i \theta}\right)\right|$ has radial limit 1 for almost all $e^{i \theta}$ on $|z|=1$ will be called of class $(U)$ in $|z|<1$.

THEOREM 1. If $f(z)$ is analytic in $|z|<1$ and of class (LP), then every complex number (including $\infty$ ) which satisfies (1) is an asymptotic value of $f(z)$.

Assume that a finite $\zeta$ satisfying (1) is not an asymptotic value of $f(z)$; clearly, we need not consider the case that $\zeta=0$. Since $f(z)$ is of class (LP), the function $\phi(z)=\zeta^{-1}[\zeta-f(z)]$ has radial limit 1 almost everywhere and is then of class $(U)$ in $|z|<1$. Because the $\zeta$-points of $f(z)$ satisfy (1), we may write $\phi(z)=B_{\zeta}(z) F(z)$, where $B_{\zeta}(z)$ is a Blaschke product extended over the zeros of $\phi(z)$. It is well known $[7$, p. 94] that the radial limits of a Blaschke product exist and have modulus 1 almost everywhere on $|z|=1$. From this it follows that $F(z)$ is of class (U) without zeros in $|z|<1$. It is then a conse- 
quence of Lemma 1 that, unless $F(z)$ is identically constant, $F(z)$ must admit either 0 or $\infty$ as an aymptotic value. We remark first that $F(z)$ cannot be constant; for if $\phi(z)$ reduces to a Blaschke product whose radial limit is 1 almost everywhere, the Riesz-Nevanlinna theorem shows that $\phi(z)$, and consequently $f(z)$, is constant. We assert next that 0 must be an asymptotic value of $F(z)$; otherwise $|F(z)|>1$ in $|z|<1$, so that $\phi(z)$ could be expressed as the quotient of two bounded functions in $|z|<1$, i.e., $\phi(z)$ would be of bounded characteristic in $|z|<1$. Again, by the Riesz-Nevanlinna theorem, $\phi(z)$ would be constant in $|z|<1$. Since zero must now be an asymptotic value of $F(z)$, and since $B_{\zeta}(z)$ is bounded, $\phi(z)$ must admit zero as an asymptotic value, so that $\zeta$ is an asymptotic value of $f(z)$.

To show that $f(z)$ admits $\infty$ as an asymptotic value, ${ }^{1}$ we remark that the function $g(z)=e^{f(z)}$ is of class (U) without zeros in $|z|<1$. Applying Lemma 1 to $g(z)$, we see that, since $g(z)$ is not constant, $g(z)$ admits either 0 or $\infty$ as an asymptotic value, so that there exists at least one path $\mathcal{L}$ terminating at some point $e^{i \theta_{0}}$ of $|z|=1$ along which $f(z) \rightarrow \infty$. Thus Theorem 1 is proved.

We remark that Theorem 1 is related to a recent result of Cartwright and Collingwood [2, p. 112], the added hypothesis that $f(z)$ be of class (LP) in $|z|<1$ allowing us to obtain a stronger conclusion to part of Theorem 9 of their paper.

2. In order to determine how frequently a function of class (LP) admits as an asymptotic value a complex number $\zeta$ satisfying (1), it will be necessary to use a form of the Schwarz reflection principle developed recently in [4]. We summarize this principle as a lemma.

Lemma 2. Let $f(z)$ be meromorphic in $|z|<1$ and let $A$ be the arc $0 \leqq \theta_{1}<\theta<\theta_{2}<2 \pi$. Let there exist an $\epsilon>0$ such that $f(z)$ has no zeros or poles in the region $0<1-|z|<\epsilon, \theta_{1}<\arg z<\theta_{2}$, and let the modulus $\left|f\left(r e^{i \theta}\right)\right|$ have radial limit 1 for almost all $e^{i \theta}$ on $A$. Then a necessary and sufficient condition that $f(z)$ may be continued analytically across the arc $A$ by means of the reflection principle $f(\bar{z})=1 / \bar{f}(1 / z)$ is that $f(z)$ admit neither 0 nor $\infty$ as an asymptotic value on $A$.

We proceed now to the principal result of this paper.

THEOREM 2. Let $f(z)$ be analytic in $|z|<1$ and of class (LP) on an arc $\alpha<\theta<\beta$ of $|z|=1$. Let $A$ be an arbitrary sub-arc of $(\alpha, \beta)$ and $\zeta$ an arbitrary complex number (including $\infty$ ). If there is a neighborhood

1 The method of proof of the previous paragraph shows also that $\infty$ is an asymptotic value of $F(z)$, but the presence of the factor $B_{\zeta}(z)$ precludes an immediate inference that $\infty$ is an asymptotic value of $\phi(z)$, and, consequently, of $f(z)$. 
$V\left(e^{i 0} \Delta\right) \cap K$ of the midpoint $e^{i \theta \Delta}$ of $A$ in which the $\zeta$-points of $f(z)$ satisfy (1), then there exists a point $e^{i \theta_{0}}$ on that part of $A$ which bounds $V \cap K$, and a Jordan arc $\mathcal{L}$ of $|z|<1$ terminating at $e^{i 0_{0}}$ such that $f(z) \rightarrow \zeta$ as $z \rightarrow e^{i \theta_{0}}$ along $\mathcal{L}$.

Let us suppose that there exists an $\operatorname{arc} A\left(\theta_{1}<\theta<\theta_{2}\right)$, with midpoint $e^{i \theta \Delta}$ and contained in $(\alpha, \beta)$, and a complex number $\zeta$ satisfying (1) in some neighborhood $V\left(e^{i \theta} \Lambda\right) \cap K$ which $f(z)$ does not admit as an asymptotic value on that subarc $B$ of $A$ which bounds $V\left(e^{i \theta A}\right) \cap K$. The case $\zeta=0$ being trivial, we may suppose that $\zeta$ is not 0 , and, for the moment, not $\infty$. Since $f(z)$ is of class (LP) on $A$, the function $\phi(z)=\zeta^{-1}[\zeta-f(z)]$ is analytic in $|z|<1$ and has radial limit 1 for almost all $e^{i \theta}$ on $A$. Since the $\zeta$-points of $f(z)$ satisfy (1) in $V\left(e^{i \theta} \Lambda\right) \cap K$, we may write, as before, $\phi(z)=B_{5}(z) F(z)$, where $B_{5}(z)$ is a Blaschke product extended over the zeros of $\phi(z)$ in $V\left(e^{i \theta \Lambda}\right) \cap K$, and where $F(z)$ is analytic without zeros in $V\left(e^{i \theta} A\right) \cap K$. The function $F(z)$ must possess radial limit values of modulus 1 for almost all $e^{i \theta}$ on $B$. It cannot happen that $F(z)$ is the quotient of two bounded functions $V\left(e^{i \theta} \Lambda\right) \cap K$; for otherwise the Riesz-Nevanlinna theorem would imply that $\phi(z)$, and consequently $f(z)$, is identically constant. Furthermore, it is clear that no point of $B$ can be a regular point of $F(z)$. It follows from Lemma 2 that the set of singularities of $F(z)$ on $B$ (namely, all points of $B$ ) is the closure of the set of points $e^{i \theta}$ on $B$ which are the terminal points of Jordan arcs along which either $F(z)$ $\rightarrow 0$ or $F(z) \rightarrow \infty$. A simple modification of a result of Carathéodory $[1$, pp. 266-267] and the author [3, p. 251] shows that, unless $|F(z)|$ $>1$ in $V\left(e^{i \theta} \Delta\right) \cap K, F(z)$ must admit zero as an asymptotic value on $B$. Now if $|F(z)|>1$ in $V\left(e^{i \theta A}\right) \cap K$, then $\phi(z)$ is the quotient of two bounded functions in that region and, according to the corollary of the Riesz-Nevanlinna theorem, must be identically constant. Since $F(z)$ must admit 0 as an asymptotic value on $B$, the boundedness of $B_{\zeta}(z)$ implies that 0 is an asymptotic value of $\phi(z)$ on $B$, so that $\zeta$ is an asymptotic value of $f(z)$ on $B$. This contradiction proves Theorem 2 for the case that $|\zeta|<\infty$. For the case that $\zeta=\infty$, we apply Lemma 2 directly to the function $g(z)=e^{f(z)}$, thus completing the proof of Theorem 2 .

\section{REFERENCES}

1. C. Carathéodory, Zum Schwarzschen Spiegelungsprinzip, Comment. Math. Helv. vol. 19 (1946-47) pp. 263-278.

2. E. F. Collingwood and M. L. Cartwright, Boundary theorems for a function meromorphic in the unit circle, Acta Math. vol. 87 (1952) pp. 83-146.

3. A. J. Lohwater, The boundary values of a class of meromorphic functions, Duke Math. J. vol. 19 (1952) pp. 243-252. 
4. - Les valeurs asymptotiques de quelques fonctions meromorphes dans le cercle-unite, C. R. Acad. Sci. Paris vol. 237 (1953) pp. 16-18.

5. N. Lusin and J. Privaloff, Sur l'unicite et la multiplicite des fonctions analytiques, Ann. Ecole Norm. (13) vol. 42 (1925) pp. 143-191.

6. R. Nevanlinna, Eindeutige analytische Funktionen, Berlin, Springer, 1936.

7. F. Riesz, Über die Randwerte einer analytischen Funktion, Math. Zeit. vol. 18 (1923).

UNIVERSity OF Michigan

\section{MULTIPLICATIVE GROUPS OF ANALYTIC FUNCTIONS}

\section{WALTER RUDIN}

Let $D$ be a proper subdomain of the Riemann sphere, and let $M(D)$ be the multiplicative group of all regular single-valued analytic functions on $D$ which have no zeros in $D$. It is known [1] that the algebraic structure of the ring $R(D)$ of all regular single-valued analytic functions on $D$ determines (and is determined by) the conformal type of $D$. In this paper we ask the question: what information about $D$ does the algebraic structure of $M(D)$ give, and, conversely, which properties of $D$ determine the algebraic structure of $M(D)$ ? The answer is, briefly, that $M\left(D_{1}\right)$ and $M\left(D_{2}\right)$ are isomorphic if and only if $D_{1}$ and $D_{2}$ have the same connectivity.

Here the connectivity of $D$ is $k$ if the complement of $D$ has $k$ components, and is $\infty$ if the complement of $D$ has infinitely many (countable or power of the continuum) components. The structure of $M(D)$ is described in more detail in the theorem below.

If we associate with each $f \in M(D)$ the function $g=f /|f|$ we obtain a subgroup (isomorphic to $M(D)$ ) of the multiplicative group $C(D)$ of all continuous functions from $D$ into the unit circumference. Such functions have been studied in great detail by Eilenberg [2]. It is worth noting that our theorem is valid if we replace $M(D)$ by $C(D)$, and that the proof is essentially the same; but it seems more interesting to stay within the smaller group.

Before stating the theorem, it is convenient to define two subgroups of $M(D)$.

(1) Fix a point $z_{0} \in D$ and let $G(D)$ be the set of all $f \in M(D)$ such that $f\left(z_{0}\right)=1$. Then $M(D)$ is the direct product of $G(D)$ and the multiplicative group of the nonzero complex numbers, and $G(D)$

Presented to the Society, April 24, 1954; received by the editors March 23, 1954. 